\title{
BMJ Open Accuracy, reproducibility and costs of different laboratory assays for the monitoring of unfractionated heparin in clinical practice: a prospective evaluation study and survey among Swiss institutions
}

\author{
Susanne Bürki, ${ }^{1}$ Béatrice Brand ${ }^{2}$ Robert Escher, ${ }^{2}$ Walter A Wuillemin, ${ }^{3}$ \\ Michael Nagler ${ }^{1,4}$
}

To cite: Bürki S, Brand B, Escher R, et al. Accuracy, reproducibility and costs of different laboratory assays for the monitoring of unfractionated heparin in clinical practice: a prospective evaluation study and survey among Swiss institutions. BMJ Open 2018;8:e022943. doi:10.1136/ bmjopen-2018-022943

- Prepublication history for this paper is available online. To view these files, please visit the journal online (http://dx.doi. org/10.1136/bmjopen-2017022943).

Received 14 March 2018 Revised 25 April 2018 Accepted 14 May 2018

Check for updates

${ }^{1}$ Department of Haematology, Inselspital, Bern University Hospital, University of Bern, Bern, Switzerland

${ }^{2}$ Department of Medicine, Spital Emmental, Burgdorf, Switzerland ${ }^{3}$ Division of Haematology and Central Haematology Laboratory, Luzerner Kantonsspital, Lucerne, Switzerland

${ }^{4}$ Department for BioMedical Research, University of Bern, Bern, Switzerland

Correspondence to Dr Michael Nagler; michael.nagler@insel.ch, michael.nagler@dbmr.unibe.ch

\section{ABSTRACT}

Objectives To investigate the accuracy, reproducibility and costs of different laboratory assays for the monitoring of unfractionated heparin (UFH) in clinical practice and to study test utilisation in Switzerland.

Design Prospective evaluation study and survey among Swiss hospitals and laboratories.

Setting Secondary care hospital in rural Switzerland (evaluation study); all Swiss hospitals and laboratories (survey).

Participants All consecutive patients, monitored for treatment with UFH during two time periods, were included (May to July 2014 and January to February 2015; n=254).

Outcome measures Results of activated partial thromboplastin time (aPTT), thrombin time (TT), prothrombinase-induced clotting time (PiCT) and anti-Xa activity with respect to UFH concentration

Results Spearman's correlation coefficient $\left(r_{s}\right)$ with regard to anti-Xa activity was $0.68(95 \% \mathrm{Cl} 0.60$ to 0.75$)$ for aPTT, 0.79 (0.69 to 0.86 ) for TT and 0.94 (0.93 to 0.95 ) for PiCT. The correlation $\left(\mathrm{r}_{\mathrm{s}}\right)$ between anti-Xa activity and heparin concentration as determined by spiking plasma samples was 1.0 (1.0 to 1.0). The coefficient of variation was at most $5 \%$ for PiCT and anti-Xa activity (within-run as well as day-to-day variability). The total costs per test in Swiss Francs (SFr) were SFr23.40 for aPTT, SFr33.30 for TT, SFr15.70 for PiCT and SFr24.15 for anti-Xa activity. The various tests were employed in Swiss institutions with the following frequencies: aPTT 53.2\%, TT 21.6\%, anti-Xa activity $7.2 \%$, PiCT $1.4 \% ; 16.6 \%$ of hospitals performed more than one test.

Conclusions The accuracy and reproducibility of PiCT and anti-Xa activity for monitoring of UFH was superior, and analytical costs were equivalent to or lower than aPTT and TT.

\section{INTRODUCTION}

Monitoring unfractionated heparin (UFH) treatment is common practice. This is mainly due to the experience that the patient's
Strengths and limitations of this study

We studied important aspects of heparin monitoring comprehensively in a joint investigation and compared the accuracy of activated partial thromboplastin time, thrombin time, prothrombinase-induced clotting time and anti-Xa measurements directly.

- The study was conducted in the routine clinical practice of a rural secondary care hospital in Switzerland.

- As an important limitation, our study was conducted in one institution only.

- No clinical data were recorded and conclusions about clinical outcomes are not possible.

response to UFH, measured with laboratory tests such as the activated partial thromboplastin time (aPTT), varies greatly. ${ }^{1}$ Clinical data supporting laboratory monitoring of UFH are however scarce and the evidence is limited. Observational studies have suggested that the occurrence of thromboembolism as well as bleeding is associated with the heparin dose applied..$^{2-5}$ The occurrence of thromboembolism has been associated with lower aPTT values in small observational studies, ${ }^{67}$ and in a meta analyses of randomised controlled trials. ${ }^{89}$ Following these findings, monitoring of UFH is recommended by all scientific guidelines. ${ }^{10-12}$

There is however no consensus regarding the optimal laboratory test to be chosen in clinical practice. ${ }^{11-13}$ aPTT is suggested most often but the application of aPTT has important limitations. There is significant variability in sensitivity among different reagents and analysers ${ }^{12}$ and the aPTT measurement is influenced by many factors that vary in the 
critically ill. ${ }^{10}$ The thrombin time (TT), a clotting test performed by adding a known concentration of bovine thrombin to patient plasma, is used by a number of laboratories ${ }^{11}$ because TT is not influenced by factor VIII and other conditions associated with a prolonged baseline aPTT. However, the dose-response curve is not linear and at least two thrombin concentrations are needed to cover the full spectrum of drug concentration. ${ }^{11} 1415$ Chromogenic anti-Xa activity assays were established ${ }^{16}{ }^{17}$ but the reproducibility between the different assays is regarded as limited. ${ }^{18}$ In addition, the prothrombinase-induced clotting time (PiCT) assay was developed to facilitate the monitoring of different anticoagulants. ${ }^{19}$ The activated clotting time (ACT) is a rapid and easy-to-use point-ofcare test widely applied in catheterisation labs and in cardiac surgery. ${ }^{20}{ }^{21}$ It is however influenced by many factors other than heparin concentration. ${ }^{2021}$ Overall, the clinical and laboratory data for the above-mentioned tests are limited and there is still no consensus regarding the optimal test to be used in clinical practice.

To contribute to the discussion and help resolve the uncertainties associated with the various UFH tests, we have conducted an evaluation study to investigate the diagnostic accuracy, reproducibility and costs of the various assays in clinical practice in a primary care hospital in rural Switzerland. In addition, we conducted a survey among healthcare institutions to study utilisation of assays in Switzerland.

\section{METHODS}

\section{Study design, setting and population}

This prospective evaluation study was conducted in a secondary care hospital in rural Switzerland (Spital Emmental, Burgdorf, Switzerland) and all tests were performed during routine clinical practice. All consecutive patients, monitored for treatment with UFH during two time periods, were included (May to July 2014 and January to February 2015) and residual plasma samples were assessed with aPTT, TT, anti-Xa activity and PiCT. We did not apply ACT because it is used in special situations only (catheter lab, cardiac surgery) and not in a typical secondary care hospital. Patients were treated in hospital for a variety of medical and surgical diseases, and represent the typical mixed population of a secondary care hospital. The flow of the patients is shown in figure 1 and in line with official regulations no identifying or clinical data were retrieved. The laboratory of the hospital is accredited by the Swiss Accreditation Service.

\section{Patient involvement}

We did not involve patients at an early stage of this research project.

\section{Collection and handling of blood samples}

A standardised protocol for taking blood was implemented at Spital Emmental to ensure direct comparisons and to prevent preanalytical effects. ${ }^{22}$ Plastic syringes containing $1 \mathrm{~mL}$ trisodium citrate $(0.106 \mathrm{~mol} / \mathrm{L})$ per $9 \mathrm{~mL}$ of blood were used (Monovette, Sarstedt, Nümbrecht, Germany). For practical reasons, samples were all frozen prior to conducting the various laboratory assays in the first group of patients in the study. Centrifuged plasma samples (1600 $\mathrm{g}$ for $10 \mathrm{~min}$ ) were snap-frozen at $-35^{\circ} \mathrm{C}$, and analysed in batches. Analyses were performed within 4 weeks. For the second batch of patients, blood was taken but not frozen. The various laboratory assays were done within 4 hours of blood withdrawal (figure 1).

\section{Determination of laboratory assays}

aPTT was determined using HemosIL aPTT-SP (silicate activator; Instrumentation Laboratory, Bedford, Massachusetts, USA). Fifty microlitres of patient plasma were mixed with $50 \mu \mathrm{l}$ of aPTT-SP. TT is a coagulation test measuring the time to fibrin formation after adding thrombin. ${ }^{23}$ Because of a high sensitivity for heparin, two thrombin concentrations are necessary to cover the full spectrum of heparin concentration. We used $3 \mathrm{U} / \mathrm{mL}$ (TT 1) and $7.5 \mathrm{U} / \mathrm{mL}$ (TT 2) of bovine thrombin (HemosIL TT; Instrumentation Laboratory, Bedford, Massachusetts, USA). TT 2 was determined in case of an unclottable TT 1 corresponding to a high concentration of heparin. Anti-Xa activity was determined using HemosIL liquid anti-Xa (Instrumentation Laboratory, Bedford, Massachusetts, USA), calibrated with HemosIL heparin calibrators. PiCT was measured using Pefakit PiCT (DSM Pentapharm, Basel, Switzerland). The test has been described in detail elsewhere. ${ }^{19}{ }^{24}$ Briefly, $50 \mu \mathrm{L}$ of patient plasma was incubated for $180 \mathrm{~s}$ with an activator containing activated factor Xa, phospholipids and Russell's viper venom. Coagulation was initiated with calcium chloride and clotting time (s) measured. All analyses were performed on an ACL TOP 300 (Instrumentation Laboratory, Bedford, Massachusetts, USA).

\section{Determination of the anti-Xa calibration curve}

Scientific guidelines recommend calibration of laboratory tests used for heparin monitoring against plasma heparin concentration using methods such as anti-Xa activity or protamine titration. ${ }^{1325}{ }^{26}$ Whereas the accuracy of aPTT, TT and PiCT was investigated in relation to the anti-Xa activity, we demonstrated the accuracy of the anti-Xa activity assay with regard to the heparin concentration as determined using a titration study. A plasma pool was generated by mixing $5 \mathrm{~mL}$ of citrated plasma from 20 healthy volunteers demonstrated to have aPTT, PT, TT and fibrinogen concentrations within the reference range. A stock solution was prepared by adding $0.1 \mathrm{~mL}$ of UFH (HEPARIN Fresenius, 5,000 IU/ $\mathrm{mL}$, Fresenius Medical Care, Oberdorf, Switzerland) to $9.9 \mathrm{~mL}$ of plasma pool, resulting in a concentration of 50 $\mathrm{IU} / \mathrm{mL}$. This stock solution was diluted by 1:100 to obtain a working solution of $0.5 \mathrm{IE} / \mathrm{mL}$. Decreasing volumes of the working solutions were added to increasing volumes of the plasma pool, resulting in samples with a heparin concentration ranging between $0.00 \mathrm{IU} / \mathrm{mL}$ and $1.0 \mathrm{IU} /$ 


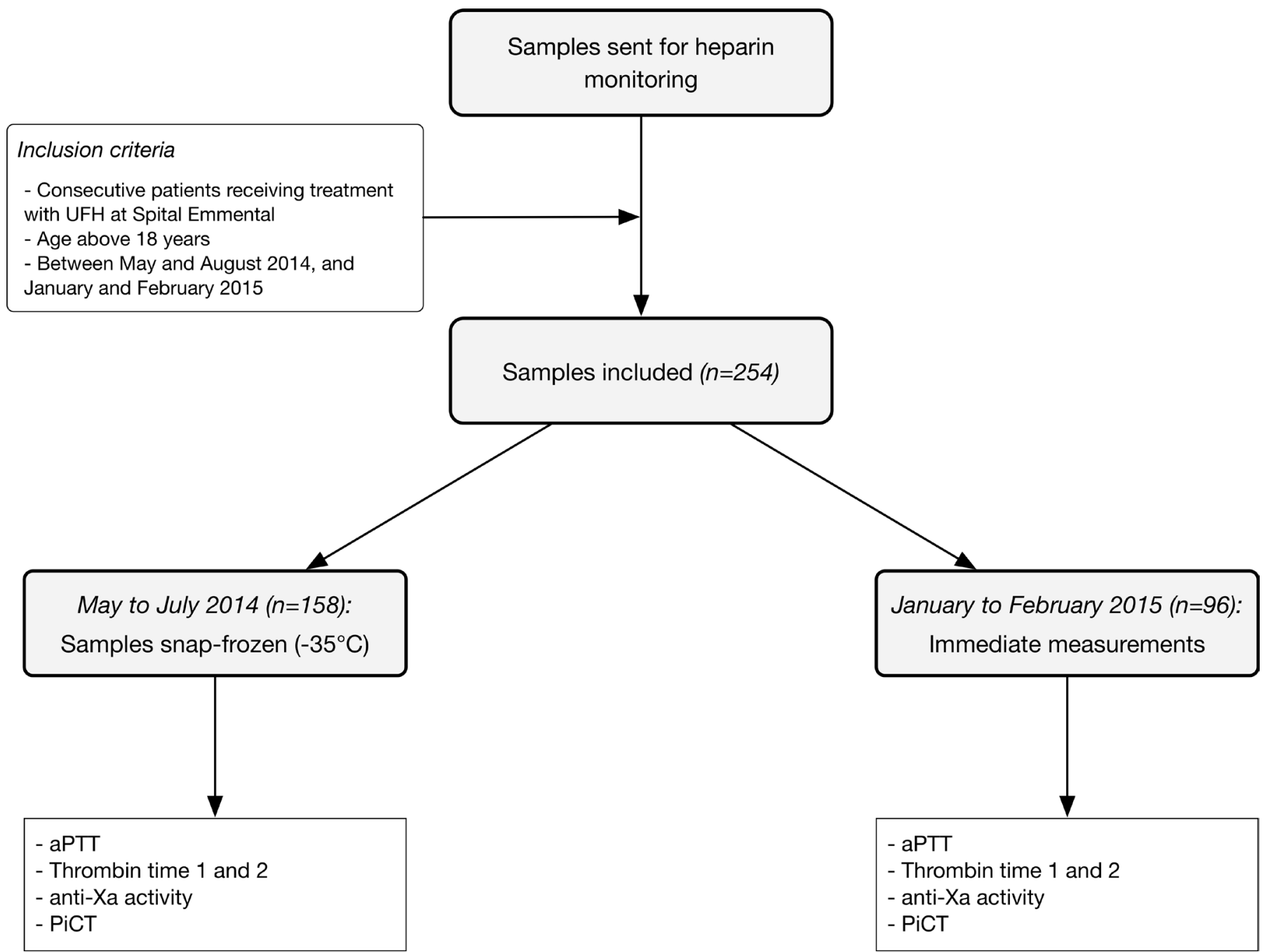

Figure 1 Flow of the samples. aPTT, activated partial thromboplastin time; PiCT, prothrombinase-induced clotting time; UFH, unfractionated heparin.

mL. Anti-Xa activity was determined and a calibration curve created.

\section{Reproducibility}

We investigated the reproducibility of the above-mentioned laboratory assays by repeating the measurements one after another in one run using two samples, one with normal results and one with abnormal results (within-run variability). The day-to-day variability was studied by repeating the measurements using frozen aliquots of two samples (abnormal and normal, respectively).

\section{Costs}

We calculated the analytical cost of the various assays by taking the following factors into consideration: reagents, controls, calibrators, consumables and time taken. Labour costs were estimated according to the average wage of a qualified technician in Switzerland (SFr42, per hour). We considered the determination of TT 2 in $48 \%$ of cases (as done in our investigation) corresponding to a high concentration of heparin. For the determination of the time taken, we included the centrifugation time, time for analysis, as well as validation. Costs were based on the Swiss healthcare system.

\section{Monitoring of UFH in clinical practice-a survey among Swiss healthcare institutions}

Two sources were used to identify the Swiss healthcare institutions, which monitor UFH. First, the official list of hospitals, published by the Federal Office of Public Health (Federal Office of Public Health, Key figures for Swiss hospitals (Internet). 2013 (cited 01 November 2016). Available from: https://www.bag.admin.ch/bag/ en/home/service/zahlen-fakten/zahlen-fakten-zu-spitaelern/kennzahlen-der-schweizer-spitaeler.html), and second, a list of laboratories providing training for laboratory specialists, issued by the Swiss Society of Medical Laboratories (FAMH) (The medical laboratories of Switzerland (FAMH), FAMH Weiterbildungsstätten (Internet). 2016 (cited 01 November 2016). Available from: https://www.famh.ch/weiterbildung-fortbildung/ weiterbildung/famh-weiterbildungsstaetten/). The downloaded information was checked and duplicate 
Table 1 Accuracy of the various laboratory tests for heparin monitoring in clinical practice

\begin{tabular}{|c|c|c|c|c|c|}
\hline \multirow[b]{2}{*}{ Assay } & \multirow[b]{2}{*}{ Observations } & \multicolumn{3}{|c|}{ Spearman's correlation coefficient $(95 \% \mathrm{Cl})$} & \multirow[t]{2}{*}{$\begin{array}{l}\text { Regression } \\
\text { coefficients }^{*}\end{array}$} \\
\hline & & All samples & Unfrozen only & Frozen only & \\
\hline aPTT† & 193 & $0.68(0.60$ to 0.75$)$ & 0.62 (0.36 to 0.79$)$ & 0.71 (0.63 to 0.78$)$ & $Y=158.6^{*} X+22.3$ \\
\hline TT 1 (low)† & 163 & 0.73 (0.65 to 0.79$)$ & 0.79 (0.66 to 0.87$)$ & 0.71 (0.60 to 0.79$)$ & $Y=173.7^{\star} X+1.86$ \\
\hline Tा 2 (high)† & 87 & 0.79 (0.69 to 0.86$)$ & 0.72 (0.54 to 0.84$)$ & 0.85 (0.74 to 0.92$)$ & $Y=94.0^{*} X-24.2$ \\
\hline $\mathrm{PiCT} \dagger$ & 254 & 0.94 (0.93 to 0.95$)$ & 0.96 (0.94 to 0.97$)$ & 0.93 (0.91 to 0.95$)$ & $Y=189.5^{*} X+24.6$ \\
\hline Anti-Xał & 14 & $1.0(95 \% \mathrm{Cl} 1.0$ to 1.0$)$ & N/A & N/A & $Y=1.005^{\star} X+0.03977$ \\
\hline
\end{tabular}

*Linear regression according to the Deming procedure was used.

†With regard to anti-Xa activity.

¥With regard to a calibration curve derived from dilution studies using heparin-spiked plasma samples.

aPTT, activated partial thromboplastin time; PiCT, prothrombinase-induced clotting time; $\Pi \mathrm{T}$, thrombin time 1 ( $3 \mathrm{U} / \mathrm{mL}$ bovine thrombin), $\mathrm{TT}$

2, thrombin time 2 (7.5 U/mL bovine thrombin).

entries were removed. A structured telephone interview was then conducted with the senior technician at each laboratory and the details recorded regarding the laboratory tests used to monitor UFH.

\section{Statistical analysis}

Numbers and percentages were reported as appropriate. We calculated the Spearman correlation coefficients to describe associations between results of aPTT, TT, PiCT, anti-Xa activity and heparin concentration. A linear regression according to the Deming procedure was additionally calculated. The coefficient of variation (CV) was used to describe within-run as well as day-to-day variability (mean is reported). No explicit power calculation has been done. Analyses were performed using the Stata V.14 statistics software package (Stata Statistical Software: Release 14; StataCorp, 2014; College Station, Texas, USA); figures were created using Prism V.6 (GraphPad Software, La Jolla California USA).

\section{RESULTS}

\section{Patient samples}

Samples from 254 patients receiving treatment with UFH between May 2014 and February 2015 were evaluated. The flow of the samples is shown in figure 1. Between May to July 2014, blood samples were snap-frozen and analysed in batches; between January to February 2015, samples were analysed within 4 hours following the taking of blood.

\section{Accuracy}

The correlation between the different laboratory assays and anti-Xa activity is shown in table 1 which shows the values associated with all samples as well as the outcomes for the frozen or unfrozen batches. Figure 2 illustrates the distribution of the results and shows the regression line according to the Deming procedure. In relation to anti-Xa activity, the overall Spearman correlation coefficient $\left(\mathrm{r}_{\mathrm{s}}\right)$ was $0.68(95 \%$ CI 0.60 to 0.75$)$ for aPTT, 0.79 (0.69 to 0.86) for TT and 0.94 (0.93 to 0.95) for PiCT. Even though differences were noted between the frozen and unfrozen samples, the overall findings remain the same. Correlation $\left(r_{s}\right)$ between anti-Xa activity and heparin concentration was 1.0 (95\% CI 1.0 to 1.0 ; figure 3).

\section{Reproducibility}

Measures of reproducibility are reported in table 2 . Within-run variability, as determined by CV, was $5.7 \%$ for aPTT, $14.7 \%$ for TT (high concentration), $2.5 \%$ for PiCT and $3.4 \%$ for anti-Xa. Day-to-day variability was $3.7 \%$ for aPTT, $6.1 \%$ for TT, 1.1 for PiCT and 5.0 for anti-Xa activity.
A

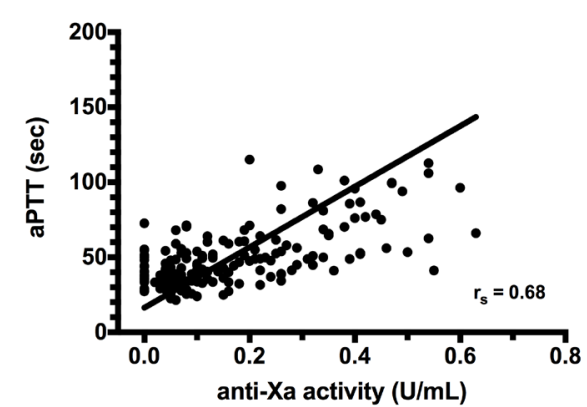

B

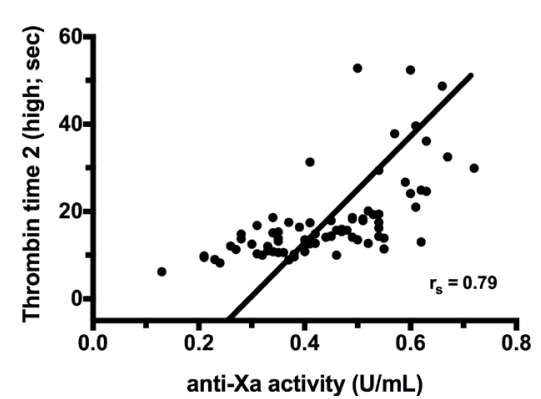

C

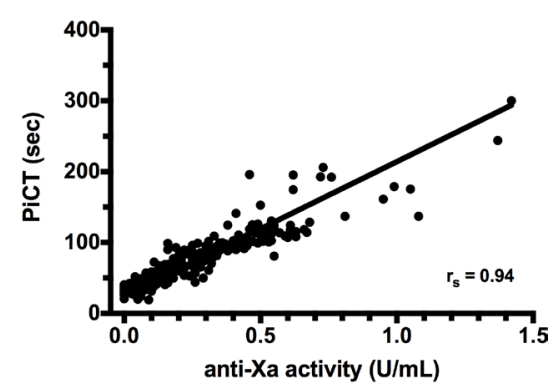

Figure 2 Accuracy of different assays for heparin monitoring in clinical practice. (A) Activated partial thromboplastin time (aPTT), (B) thrombin time (high concentration), (C) prothrombinase-induced clotting time (PiCT). Scattergram with regression curves (Deming procedure) and correlation coefficients are shown. 


\section{Anti-Xa activity}

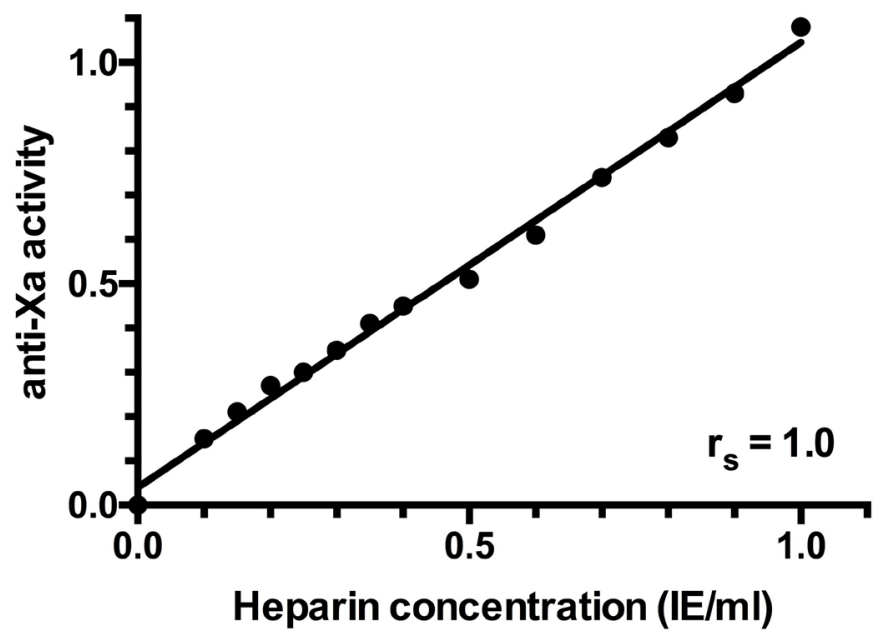

Figure 3 Anti-Xa calibration curve according to titration studies using unfractionated heparin.

\section{Time and cost estimates}

The overall costs for each of the assays in Swiss Francs (SFr) were found to be SFr23.40 (aPTT), SFr33.30 (TT), SFr15.70 (PiCT) and SFr24.15 (anti-Xa activity). The times and costs required for each assay type were calculated are reported in table 3 with PiCT costing the least.

\section{Monitoring of UFH in Switzerland}

One hundred and thirty-nine Swiss institutions were identified which conduct heparin monitoring in clinical practice. The vast majority of institutions implemented aPTT, TT or both assays (84.9\%). Of the institutions $53.2 \%$ relied exclusively on aPTT, $13.7 \%$ on anti-Xa activity and PiCT was used by just two institutions $(1.4 \%)$. Further details are provided in table 4 .

\section{DISCUSSION}

We found that concordance of laboratory measurements with heparin concentration was better for anti-Xa activity and PiCT compared with aPTT and TT. Reproducibility of anti-Xa and PiCT was at least comparable to aPTT. Analytical costs of PiCT were lower compared with aPTT. The majority of Swiss institutions conduct aPTT or TT for heparin monitoring.
Table 3 Laboratory tests for heparin monitoring in clinical practice: time and cost

\begin{tabular}{lll}
\hline Test & $\begin{array}{l}\text { Time required for } \\
\text { measurement }^{*}(\mathbf{m i n})\end{array}$ & $\begin{array}{l}\text { Cost of the } \\
\text { analysis† (in SFr) }\end{array}$ \\
\hline aPTT & 21 & 23.40 \\
TT & $28 \ddagger$ & $33.30 \ddagger$ \\
PiCT & 20 & 15.70 \\
Anti-Xa & 17 & 24.15
\end{tabular}

*Including centrifugation, analysis and validation.

†Including reagents, calibrators, quality control as well as work expended; labour costs were estimated according to the average wage of a qualified technician in Switzerland (SFr42 per hour). fDetermination of thrombin time 2 (high concentration) in $48 \%$ of the cases was considered.

aPTT, activated partial thromboplastin time; PiCT, prothrombinaseinduced clotting time; $\pi$, thrombin time.

Concordance of aPTT with heparin concentration is generally regarded as poor. ${ }^{26-28}$ The correlation coefficients we found are in line with previous studies. Bounameaux et al, ${ }^{14}$ in a study of 63 plasma samples, determined that Pearson's correlation coefficient was 0.85 in case of TT and 0.67 in case of aPTT. In another analysis of 49 patients, ${ }^{15}$ a coefficient of determination $\left(\mathrm{r}^{2}\right)$ of 0.63 for aPTT and 0.74 for TT was found. A multicentre study in 377 patients returned a correlation of 0.76 for aPTT with anti-Xa levels. ${ }^{24}$ A limited agreement has been observed in a prospective study in 569 patients. ${ }^{29}$ Several studies have demonstrated a dose-dependent prolongation of TT. ${ }^{12} 14$ In In agreement with our results, alignment with heparin concentration was better with aPTT. Correlation of PiCT with anti-Xa levels was $r=0.88$ in a multicentre study in 377 patients. ${ }^{24}$ With regard to the anti-Xa activity, one small randomised controlled trial compared anti-Xa activity assay monitoring with aPTT and reported fewer complications in the case of anti-Xa. ${ }^{30}$

The strength of our investigation is that we have studied several important aspects of heparin monitoring in clinical practice in Switzerland in a joint investigation and compared the accuracy of aPTT, TT, PiCT and anti-Xa measurements directly.

Our study was conducted in one institution only. Therefore, we cannot exclude that results could be different if analyses were conducted in other laboratories. This

Table 2 Reproducibility of different laboratory tests for heparin monitoring in clinical practice

\begin{tabular}{|c|c|c|c|c|}
\hline \multirow[b]{2}{*}{ Assay } & \multirow{2}{*}{$\begin{array}{l}\text { Within-run variability } \\
\text { CV }\end{array}$} & \multirow[t]{2}{*}{$\begin{array}{l}\text { Number of } \\
\text { measurements }\end{array}$} & \multirow{2}{*}{$\begin{array}{l}\text { Day-to-day variability } \\
\text { CV }\end{array}$} & \multirow{2}{*}{$\begin{array}{l}\text { Number of } \\
\text { measurements }\end{array}$} \\
\hline & & & & \\
\hline aPTा & $5.7 \%$ & 10 & $3.7 \%$ & 15 \\
\hline TT 2 (low) & $14.7 \%$ & 10 & $6.1 \%$ & 15 \\
\hline РiCT & $2.5 \%$ & 10 & $1.1 \%$ & 15 \\
\hline Anti-Xa & $3.4 \%$ & 10 & $5.0 \%$ & 15 \\
\hline
\end{tabular}

aPTT, activated partial thromboplastin time; CV, coefficient of variation (\%); PiCT, prothrombinase-induced clotting time; TT 2, thrombin time 2 (7.5 $\mathrm{U} / \mathrm{mL}$ bovine thrombin). 
Table 4 Laboratory tests used for heparin monitoring in clinical practice: a survey across healthcare institutions in Switzerland

\begin{tabular}{lc}
\hline Laboratory test used & Frequency, numbers (\%) \\
\hline aPTT & $74(53.2)$ \\
TT & $30(21.6)$ \\
Anti-Xa & $10(7.2)$ \\
PiCT & $2(1.4)$ \\
aPTT and anti-Xa & $5(3.6)$ \\
aPTT, and TT & $14(10.1)$ \\
aPTT, TT and anti-Xa & $4(2.9)$ \\
\hline
\end{tabular}

Two sources were used to identify institutions and contacts for interviews: (1) all Swiss hospitals providing inpatient services and (2) all laboratories accredited to the Swiss Accreditation Service providing training for laboratory specialists.

aPTT, activated partial thromboplastin time; PiCT, prothrombinaseinduced clotting time; $\mathrm{TT}$, thrombin time.

issue should be studied in future investigations. Second, samples were treated differently between the two phases of our study; the first batch of samples were frozen prior to conducting the laboratory assays for practical reasons, and the second batch were not frozen. Although the results between these two batches of samples were found to differ (table 1), the differences found between the various assays nevertheless remained the same. And thirdly, correlation coefficient is not an ideal accuracy measure in case of TT, because correlation with heparin is not linear and two concentrations are needed to monitor UFH. Finally, there were no clinical data retrieved. Therefore, conclusions about clinical outcomes are not possible.

In conclusion, we have found that performance of PiCT and anti-Xa activity for heparin monitoring is superior to TT and aPTT in a single centre evaluation study in a secondary care hospital in Switzerland.

Acknowledgements The authors thank the laboratory technicians of Spital Emmental for their support.

Contributors SB contributed to study design, conducted laboratory tests, conducted the survey, analysed the data and wrote the manuscript. BB contributed to study design and conducted laboratory tests. RE contributed to study design, provided infrastructure and reagents, and reviewed the statistical analysis. WAW contributed to study design and revised the statistical analysis. MN developed the study design and the statistical analysis plan, conducted the statistical analysis, and wrote the manuscript. All authors have reviewed and approved the final manuscript.

Funding This research received no specific grant from any funding agency in the public, commercial or not-for-profit sectors.

Competing interests MN has received research grants or lecture fees from Bayer, Axonlab and Roche Diagnostics.

Patient consent Not required.

Ethics approval The protocol was approved by the local ethics committee (Kantonale Ethikkommission Bern; 18 March 2014).

Provenance and peer review Not commissioned; externally peer reviewed.

Data sharing statement All requests should be directed to the corresponding author.

Open access This is an Open Access article distributed in accordance with the Creative Commons Attribution Non Commercial (CC BY-NC 4.0) license, which permits others to distribute, remix, adapt, build upon this work non-commercially, and license their derivative works on different terms, provided the original work is properly cited and the use is non-commercial. See: http://creativecommons.org/ licenses/by-nc/4.0/

(c) Article author(s) (or their employer(s) unless otherwise stated in the text of the article) 2018. All rights reserved. No commercial use is permitted unless otherwise expressly granted.

\section{REFERENCES}

1. Hirsh J, Anand SS, Halperin JL, et al. Guide to anticoagulant therapy: Heparin : a statement for healthcare professionals from the American Heart Association. Circulation 2001;103:2994-3018.

2. Raschke RA, Reilly BM, Guidry JR, et al. The weight-based heparin dosing nomogram compared with a "standard care" nomogram. A randomized controlled trial. Ann Intern Med 1993;119:874-81.

3. Raschke RA, Gollihare B, Peirce JC. The effectiveness of implementing the weight-based heparin nomogram as a practice guideline. Arch Intern Med 1996;156:1645-9.

4. Investigators E. EPILOG Investigators. Platelet glycoprotein Ilb/ Illa receptor blockade and low-dose heparin during percutaneous coronary revascularization. N Engl J Med 1997;336:1689-96.

5. Morabia A. Heparin doses and major bleedings. Lancet 1986;1:1278-9

6. Basu D, Gallus A, Hirsh J, et al. A prospective study of the value of monitoring heparin treatment with the activated partial thromboplastin time. N Engl J Med 1972:287:324-7.

7. Kaplan K, Davison R, Parker M, et al. Role of heparin after intravenous thrombolytic therapy for acute myocardial infarction. Am $J$ Cardiol 1987:59:241-4.

8. Hull RD, Raskob GE, Hirsh J, et al. Continuous intravenous heparin compared with intermittent subcutaneous heparin in the initial treatment of proximal-vein thrombosis. N Engl J Med 1986;315:1109-14.

9. Turpie AG, Robinson JG, Doyle DJ, et al. Comparison of high-dose with low-dose subcutaneous heparin to prevent left ventricular mural thrombosis in patients with acute transmural anterior myocardial infarction. N Engl J Med 1989;320:352-7.

10. Garcia DA, Baglin TP, Weitz JI, et alAmerican College of Chest Physicians Evidence-Based Clinical Practice GuidelinesChest 2012.

11. Olson JD, Arkin CF, Brandt JT, et al. College of American Pathologists Conference XXXI on laboratory monitoring of anticoagulant therapy: laboratory monitoring of unfractionated heparin therapy. Arch Pathol Lab Med 1998;122:782-98.

12. Greaves M. Limitations of the laboratory monitoring of heparin therapy. Scientific and Standardization Committee Communications: on behalf of the Control of Anticoagulation Subcommittee of the Scientific and Standardization Committee of the International Society of Thrombosis and Haemostasis. Thromb Haemost 2002;87:163-4.

13. Kearon C, Akl EA, Comerota AJ, et al. American College of Chest Physicians Evidence-Based Clinical Practice Guidelines. Chest 2012;141:e419S-e96S.

14. Bounameaux $\mathrm{H}$, Marbet GA, Lämmle B, et al. Monitoring of Heparin Treatment. Comparison between Thrombin Time, Activated Partial Thromboplastin Time, and Plasma Heparin Concentration, and Analysis of the Behavior of Antithrombin III. Am J Clin Pathol 1980;74:68-73.

15. Ray MJ, Perrin EJ, Smith IR, et al. A proposed model to monitor heparin therapy using the concentrated thrombin time which allows standardisation of reagents and improved estimation of heparin concentrations. Blood Coagulation \& Fibrinolysis 1996;7:515-21.

16. Teien $A N$, Lie $M$, Abildgaard $U$. Assay of heparin in plasma using a chromogenic substrate for activated factor $\mathrm{X}$. Thromb Res 1976;8:413-6.

17. Smith ML, Wheeler KE. Weight-based heparin protocol using antifactor Xa monitoring. American Journal of Health-System Pharmacy 2010;67:371-4.

18. Lehman CM, Frank EL. Laboratory Monitoring of Heparin Therapy: Partial Thromboplastin Time or Anti-Xa Assay? Lab Med 2009:40:47-51.

19. Korte W, Jovic R, Hollenstein M, et al. The uncalibrated prothrombinase-induced clotting time test. Equally convenient but more precise than the aPTT for monitoring of unfractionated heparin Hamostaseologie 2010;30:212-6.

20. Horton S, Augustin S. Activated clotting time (ACT). Methods Mol Biol 2013:992:155-67.

21. Spinler SA, Wittkowsky AK, Nutescu EA, et al. Anticoagulation Monitoring Part 2: Unfractionated Heparin and Low-MolecularWeight Heparin. Ann Pharmacother 2005;39(7-8):1275-85. 
22. Collection C. Transport, and Processing of Blood Specimens for Testing Plasma-Based Coagulation Assays and Molecular Hemostasis Assays; Approved Guideline. Fifth Edition. Wayne, PA Clinical and Laboratory Standards Institute, 2008. CLSI document H21-A5.

23. Lillicrap D, Key N, Makris M, et al. Practical Hemostasis and Thrombosis. Oxford, UK: Wiley-Blackwell, 2009.

24. Brisset AC, Ferrández A, Krause M, et al. The PiCT test is a reliable alternative to the activated partial thromboplastin time in unfractionated heparin therapy management: results from a multicenter study. J Thromb Haemost 2016;14:2187-93.

25. Smythe MA, Priziola J, Dobesh PP, et al. Guidance for the practical management of the heparin anticoagulants in the treatment of venous thromboembolism. J Thromb Thrombolysis 2016;41:165-86.
26. Vandiver JW, Vondracek TG. Antifactor Xa levels versus activated partial thromboplastin time for monitoring unfractionated heparin. Pharmacotherapy 2012;32:546-58.

27. Kitchen S. Problems in laboratory monitoring of heparin dosage. $\mathrm{Br} \mathrm{J}$ Haematol 2000;111:397-406.

28. Eikelboom JW, Hirsh J. Monitoring unfractionated heparin with the aPTT: time for a fresh look. Thromb Haemost 2006;96:547-52.

29. Takemoto CM, Streiff MB, Shermock KM, et al. Activated partial thromboplastin time and anti-xa measurements in heparin monitoring: biochemical basis for discordance. Am J Clin Pathol 2013;139:450-6.

30. Levine MN, Hirsh J, Gent M, et al. A randomized trial comparing activated thromboplastin time with heparin assay in patients with acute venous thromboembolism requiring large daily doses of heparin. Arch Intern Med 1994;154:49-56. 